\title{
Comparação entre o concreto leve com resíduo de borracha e o concreto com EPS
}

$\frac{c \text { ABNER SANTOS BARONI SALES - Doutorando }}{\text { MURILO SANTOS PEIXOTO - DoutORAndo }}$

\section{RESUMO}

A GRANDE REDE DE CONSUMO CAPITALISTA ACARRETA CONSIGO UMA SÉRIE DE IMPACTOS NEGATIVOS AO MEIO AMBIENTE, GERANDO UMA INFINIDADE DE RESÍDUOS PERIGOSOS QUE, QUANDO NÃO GERENCIADOS ADEQUADAMENTE, PROVOCAM IMPACTOS IRREVERSíVEIS À QUALIDADE do meio ambiente e à saúde pública. Neste contexto, estima-SE QUE O DESCARTE DE PNEUS CHEGUE A CERCA DE 1,5 BILHÃO DE UNIDADES AO LONGO DO ANO, FAZENDO-SE NECESSÁRIA A BUSCA POR TECNOLOGIAS QUE INCORPOREM TAIS RESÍDUOS COMO MATÉRIA-prima para as Tecnologias de Construção Civil. Assim, este ESTUDO BUSCOU AVALIAR AS PROPRIEDADES DO REAPROVEITAMENTO

\begin{tabular}{c}
\hline LUIZ GUSTAVO PIMENTA DE PADUA - MESTRE \\
\hline PHELIPPE MENDONÇA DE PAIVA - MESTRE \\
EngeNHARIA APLICADA E SUSTENTABILIDADE IFGoIANo
\end{tabular}

DO RESÍDUO DA BORRACHA EM SUBSTITUIÇÃO DO AGREGADO GRAÚDO PARA A PRODUÇÃO DE CONCRETO LEVE, COMPARANDO-O COM O EPS (Poliestireno Expansível), um agregado leVe jÁ consolidado, BUSCANDO-SE: PROMOVER A SUBSTITUIÇÃO TOTAL DO AGREGADO; AVALIANDO AS PROPRIEDADES FRESCAS, MECÂNICAS, MASSA ESPECÍFICA E MICROESTRUTURA. O CONCRETO LEVE DESENVOLVIDO APRESENTOU PROPRIEDADES MECÂNICAS COM VALORES SUPERIORES A 8 MPA NAS COMPOSIÇÕES DESENVOLVIDAS, EVIDENCIANDO A VIABILIDADE DA APLICAÇÃO DE CONCRETO LEVE, COM AGREGADO DE RESÍDUO DE BORRACHA DE PNEU.

Palavras-chave: concreto leve, reaproveitamento, resíduo, borracha de pneu, sustentabilidade.

\section{INTRODUÇÃO}

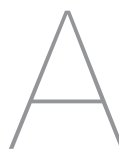

problemática do descarte inadequado de pneus lapresenta uma série de impactos negativos ao meio ambiente. Comumente é verificado o manejo inadequado deste resíduo, como sua queima ou sua disposição final inadequada em lixões ou aterros sanitários. Tais medidas colocam em risco a saúde pública e a qualidade ambiental, desrespeitando a Lei Nacional dos Resíduos Sólidos (PNRS) - Lei n 12.305/2010 (BRASIL, 2010).

Estima-se que são descartados, no mundo, cerca de 1,5 bilhões de pneus por ano. Por ser um material considerado de difícil absorção pelo ambiente, vários pesquisadores buscam alternativas para a reciclagem de forma a atenuar os impactos (KURZ et al., 2018; MOHAJERANI et al., 2020).

Uma vertente é o uso do resíduo de borracha (RB) como agregado leve para a produção de concreto, haja vista que o concreto é um dos materiais de construção que consome uma grande quantidade de recursos naturais; assim, o uso de partículas de borracha como agregado é uma abordagem que economizaria recursos naturais e reduziria o impacto gerado ao ambiente (Ll et al., 2019).

O RB apresenta baixa densidade, obtendo potencial de uso para concreto leve. De acordo com a ACl 213 R (2003), o concreto leve possui uma massa específica entre $1.120 \mathrm{~kg} / \mathrm{m}^{3}$ a $1.920 \mathrm{~kg} / \mathrm{m}^{3}$; já o concreto convencional possui massa específica compreendida entre $2.000 \mathrm{~kg} / \mathrm{m}^{3}$ e $2.800 \mathrm{~kg} / \mathrm{m}^{3}$ (NBR 8953/2015). Sendo assim, a aplicação do concreto leve reduz a carga em estruturas, uma vez que o peso próprio representa maior parte do carregamento (SAYADI et al., 2016). 


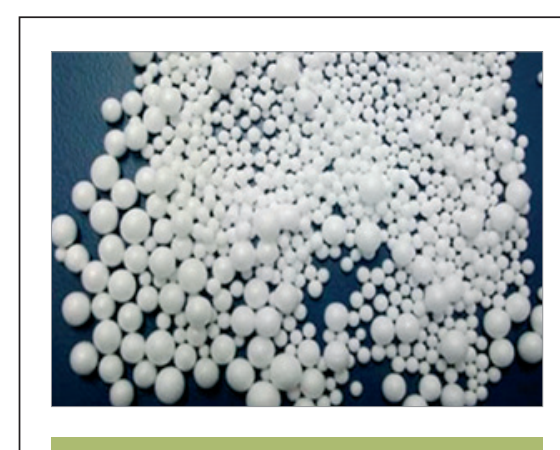

Figura 1

Pérolas de poliestireno expandido (EPS)

Fonte: Própria (2021)

O poliestireno expandido (EPS) apresenta-se como um agregado leve já consolidado para a produção de concreto leve. Xie et al. (2019) verificaram que o EPS apresenta maiores vantagens se comparado aos outros agregados leves, devido à sua baixa densidade, baixa condutividade térmica e por ter propriedades hidrofóbicas.

Portanto, este estudo visa avaliar o emprego do resíduo de borracha de pneu de como constituinte de um concreto leve e sustentável, possibili-

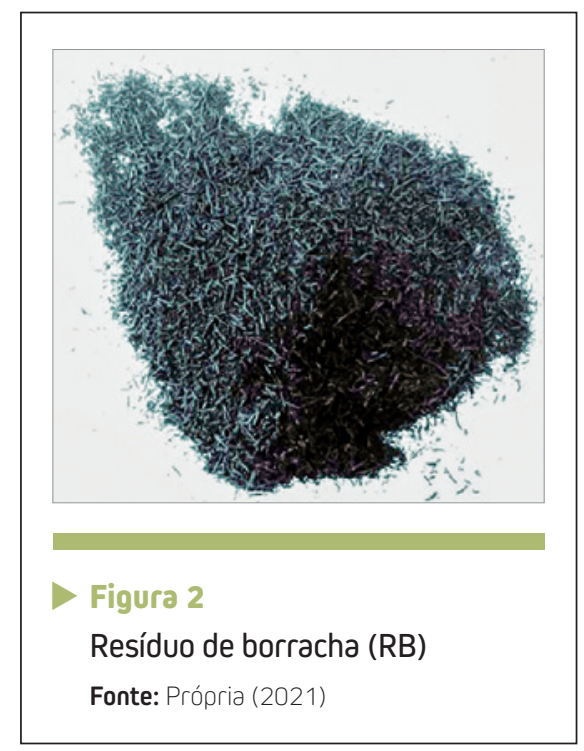

Tabela 1 - Ensaios de caracterização das matérias-primas do concreto leve mediante normas

\begin{tabular}{c|cc|}
\hline Materiais & Ensaios & Normas técnicas \\
\hline Cimento & Massa específica & NBR 16605/2017 \\
\hline Agregados graúdos & Massa específica & NBR-NM 52/2009 \\
\cline { 2 - 3 } & $\begin{array}{c}\text { Massa unitária e massa } \\
\text { unitária compactada }\end{array}$ & NBR-NM 45/2006 \\
Agregados miúdos & Massa específica e absorção & NBR-NM 53/2002 \\
\hline RB e EPS & Massa unitária & NBR-NM 45/2006 \\
& Massa específica e absorção & ACl-211.2/2004 (Apêndice A) \\
\hline
\end{tabular}

Fontes: ABNT (2002), ACI (2004), ABNT (2006), ABNT (2009), ABNT (2013), ABNT (2018)

tando a viabilidade construtiva deste comparando-o com o concreto leve de EPS através do estudo das propriedades mecânicas de ambos.

\section{METODOLOGIA}

Para o desenvolvimento do trabalho, utilizou-se os seguintes materiais:

Cimento CPV-ARI da marca CAUÊTM;

- RB coletado na empresa Recapagem Carajás;

- EPS coletado na empresa ISOCENTRO;

- Areia;

- Brita;

- Aditivo superplastificante TecFlow $50 \mathrm{~N}$ da GCPATTM.

As Figuras 1 e 2 representam respectivamente EPS e RB.

Para a caracterização dos materiais, seguiu-se as especificações das normas técnicas mediante ensaios (Tabela 1).

As composições foram definidas pelo método da norma $\mathrm{ACl}$ 211.2-98 (2004). Este trabalho adotou o méto- do massa que é o recomendado para concretos com agregados miúdos com massa específica normal e agregado leve graúdo.

As composições produzidas para o estudo constituíram-se em 3 tipos diferentes (Tabela 2), sendo uma composição referencial (CR) e o restante compostos por concreto leve. Tendo uma composição com resíduo de borracha (CRB), e uma composição com EPS (CEPS).

A partir das composições desenvolvidas, realizou-se as caracterizações do concreto baseando-se nas normas técnicas da ABNT (Tabela 3) e de normas estrangeiras. As avaliações foram realizadas em

Tabela 2 - Material utilizado em cada composição

\begin{tabular}{|ccc|}
\hline $\begin{array}{c}\text { Composição } \\
\text { de concreto }\end{array}$ & $\begin{array}{c}\text { Tipo de } \\
\text { concreto }\end{array}$ & $\begin{array}{c}\text { Agregado } \\
\text { graúdo }\end{array}$ \\
\hline CR & Referência & $\begin{array}{c}\text { Brita } \\
9,5 \mathrm{~mm}\end{array}$ \\
\hline CRB & Leve & RB \\
\hline CEPS & Leve & EPS \\
\hline
\end{tabular}

Fonte: Própria (2020) 
Tabela 3 - Ensaios da caracterização das propriedades do concreto mediante normas técnicas

\begin{tabular}{|c|c|c|}
\hline Estágio do concreto & Ensaios & Normas técnicas \\
\hline \multirow{2}{*}{ Concreto no estado fresco } & Massa específica & NBR 9833/2008b \\
\hline & Trabalhabilidade & NBR $16889 / 2020^{a}$ \\
\hline \multirow{4}{*}{ Concreto no estado endurecido } & Moldagem dos corpos de prova & NBR $5738 / 2015^{d}$ \\
\hline & Compressão axial & NBR 7215/2019f \\
\hline & Compressão diametral & NBR $7222 / 2011^{c}$ \\
\hline & Módulo elástico & NBR $8522 / 2021^{e}$ \\
\hline
\end{tabular}

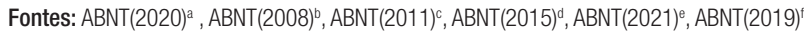

diferentes estágios do concreto: estado fresco, endurecido e pela microestrutura do concreto.

Para a determinação de absorção de água e de índice de vazios seguiu a NBR 9778 (ABNT, 2005).

\section{CARACTERIZAÇÃO DAS MATÉRIAS-PRIMAS DO CONCRETO}

A Tabela 4 apresenta os resultados dos ensaios de caracterização das matérias-primas empregadas na produção dos concretos.

O EPS apresentou valores de massa específica de 0,56\% da massa específica da brita, o justifica seu uso para alcançar a leveza do concreto, o mesmo valendo para o $\mathrm{RB}$, que, apesar de ter uma massa foram determinadas a partir da norma ACl 211.2-98 (2004) e estão presentes na Tabela 5.

\section{I Concreto no estado fresco}

Os concretos no estado fresco apresentaram diferentes resultados para massa específica (ME) e abatimento do cone de tronco (ACT), conforme elucida a Tabela 6.

Com a substituição da brita por agregados leves, foi possível reduzir significativamente a massa específica, o que se mostrou eficaz para conseguir o concreto leve. O CEPS obteve a maior redução da

\section{RESULTADOS E DISCUSSÕES}

As composições dos concretos específica superior ao EPS, ainda é apenas 19\% em relação à da brita.

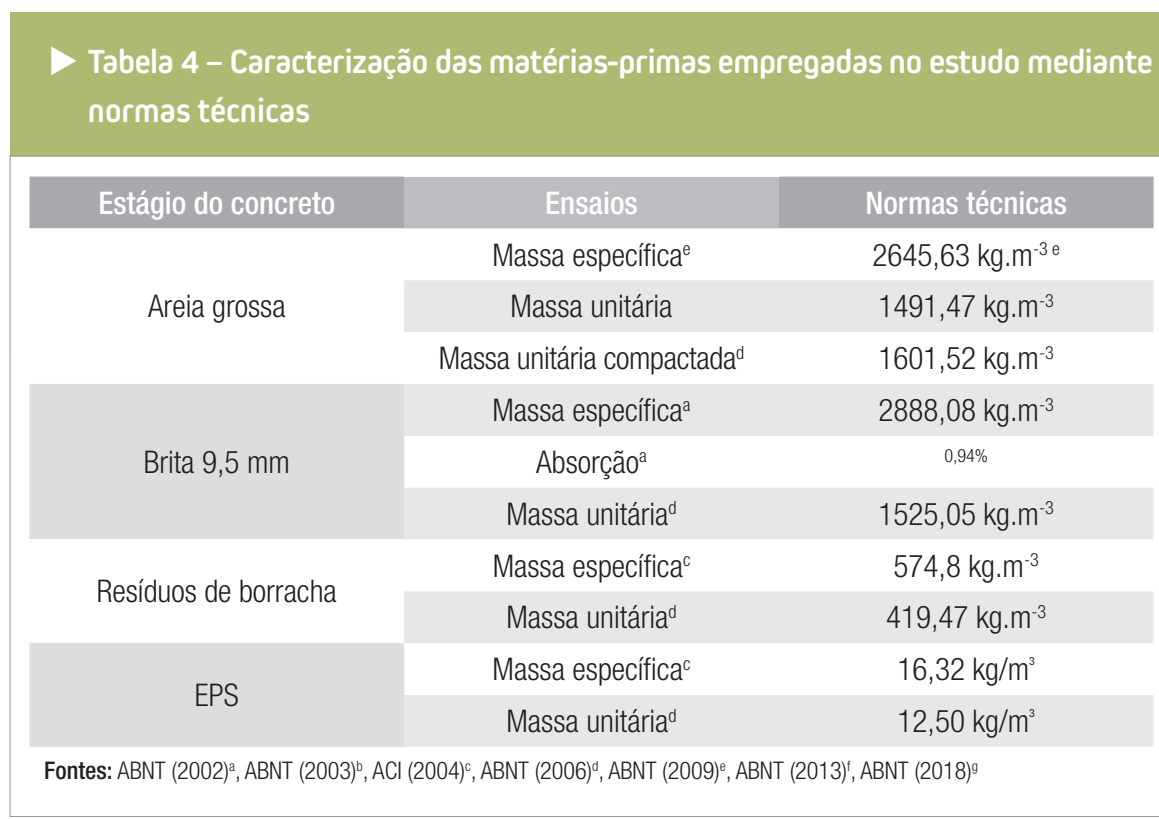

Tabela 5 - Quantitativo dos materiais utilizados na composição dos concretos

\begin{tabular}{|cccccccc|c|}
\hline Composição & $\begin{array}{c}\text { Cimento } \\
\left(\mathrm{kg} / \mathrm{m}^{3}\right)\end{array}$ & $\begin{array}{c}\text { Areia } \\
\left(\mathrm{kg} / \mathrm{m}^{3}\right)\end{array}$ & $\begin{array}{c}\text { Brita } \\
\left(\mathrm{kg} / \mathrm{m}^{3}\right)\end{array}$ & $\begin{array}{c}\text { RBP } \\
\left(\mathrm{kg} / \mathrm{m}^{3}\right)\end{array}$ & $\begin{array}{c}\text { EPS } \\
\left(\mathrm{kg} / \mathrm{m}^{3}\right)\end{array}$ & $\begin{array}{c}\text { Águla } \\
\left(\mathrm{kg} / \mathrm{m}^{3}\right)\end{array}$ & Aditivo \\
\hline CR & 557,1 & 360,04 & 1675 & 0 & 0 & 228,411 & $0 \%$ \\
\hline CRB & 557,1 & 360,04 & 0 & 333,5 & 0 & 228,411 & $0,3 \%$ \\
\hline CEPS & 557,1 & 360,04 & 0 & 0 & 7,25 & 228,411 & $0 \%$ \\
\hline Fontes: Própria (2021) & & & & & & & \\
\hline
\end{tabular}




\begin{tabular}{|c|c|c|}
\hline \multicolumn{3}{|c|}{$\begin{array}{l}\text { Tabela } 6 \text { - Resultados dos } \\
\text { concretos no estado fresco }\end{array}$} \\
\hline Composição & $\begin{array}{c}\text { ME } \\
\left(\mathrm{kg} \cdot \mathrm{m}^{-3}\right)\end{array}$ & ACT $(\mathrm{cm})$ \\
\hline $\mathrm{CR}$ & 2395 & 5,5 \\
\hline CRB & 1502 & 7 \\
\hline CEPS & 1215 & 6,5 \\
\hline Fonte: Própria (202 & & \\
\hline
\end{tabular}

sua massa específica. No entanto, o uso de borracha conseguiu uma redução superior a $800 \mathrm{~kg} \cdot \mathrm{m}^{-3}$.

Schackow et al. (2014) estudaram concreto leve com EPS, encontrando valores entre 1250 kg/m³ e 1070 kg/m³, o que demontra valores próximos ao CEPS estudado na pesquisa. Apesar do CRB ter ficado com valores maiores do que o EPS de Schackow et al. (2014), isso se dá principalmente pelo resíduo de borracha ter maior densidade, mas mesmo assim, os valores ficaram próximos aos da literatura.

No caso da trabalhabilidade, a composição com CRB apresentou uma menor trabalhabilidade, sendo necessário adicionar superplastificante para se chegar a uma consistência ideal. Assim sendo, a composiçao de CRB, sem o superplastificante, apresentou segregação

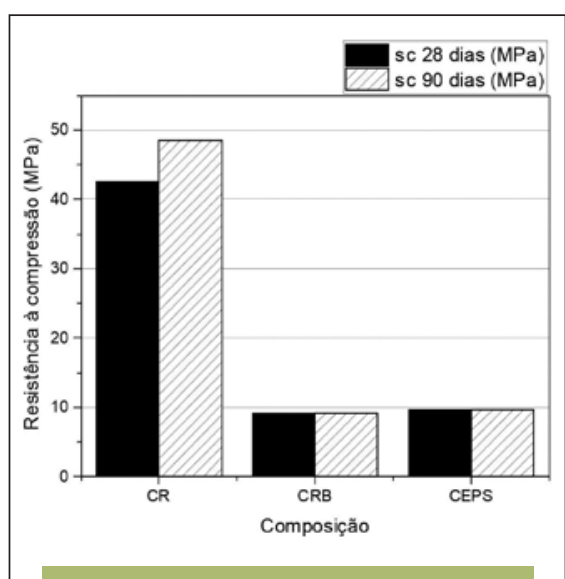

Figura 3

Resistência mecânica à compressão axial nas idades de 28 e 90 dias

Fonte: Própria (2021)

e não se uniu de forma coesa com a pasta. Porém, com a adição do superplastificante, foi possível obter uma massa coesa e bem distribuída e sem qualquer sinal de segregação.

\subsection{Concreto no estado endurecido}

No estado endurecido, os resultados foram separados em propriedades mecânicas, absorção e índices de vazios. Os resultados das propriedades mecânicas, sendo resistência à compressão axial $\left(\sigma_{c}\right)$, tração por compressão diametral $\left(\sigma_{\mathrm{R}}\right)$ e módulo de elasticidade $\left(E_{\mathrm{ci}}\right)$, foram analisadas
Tabela 7 - Resultados das propriedades mecânicas em 28 e 90 dias

\begin{tabular}{|ccccccc|c|} 
Composição & $\begin{array}{c}\sigma_{\mathrm{c}} 28 \text { dias } \\
(\mathrm{MPa})\end{array}$ & $\begin{array}{c}\sigma_{\mathrm{c}} 90 \text { dias } \\
(\mathrm{MPa})\end{array}$ & $\begin{array}{c}\sigma_{\mathrm{R}} 28 \text { dias } \\
(\mathrm{MPa})\end{array}$ & $\begin{array}{c}\sigma_{\mathrm{R}} 90 \text { dias } \\
(\mathrm{MPa})\end{array}$ & $\begin{array}{c}\mathrm{E}_{\mathrm{ci}} 28 \text { dias } \\
(\mathrm{GPa})\end{array}$ & $\begin{array}{c}\mathrm{E}_{\mathrm{ci}} 90 \text { dias } \\
(\mathrm{GPa})\end{array}$ \\
\hline CR & 42,6 & 48,52 & 3,68 & 5,25 & 38,91 & 40,93 \\
CRB & 9,08 & 9,19 & 1,01 & 1,07 & 5,37 & 5,50 \\
CEPS & 9,59 & 9,70 & 0,84 & 0,87 & 6,32 & 6,46 \\
\hline
\end{tabular}

Fonte: Própria (2021)

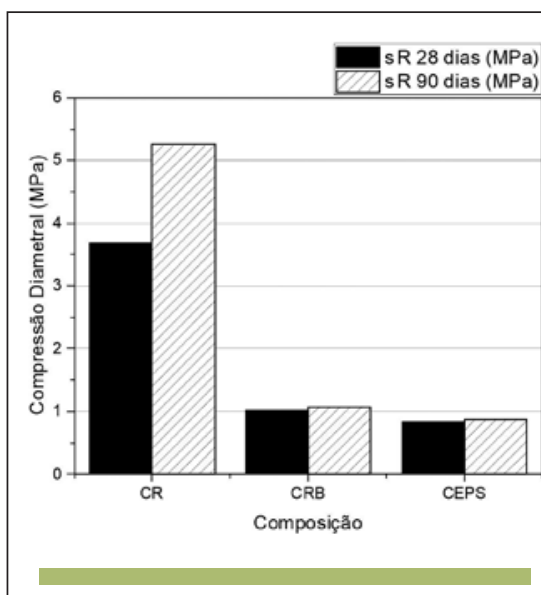

Figura 4

Resistência mecânica à tração por compressão diametral nas idades de 28 e 90 dias

Fonte: Própria (2021)

com 28 e 90 dias de cura submersa em água e seguem na Tabela 7. Os resultados de absorção e índice de vazios seguem na Tabela 7 .

As Figuras 3, 4 e 5 apresentam as propriedades mecânicas em compressão axial, compressão diametral e módulo elástico, respectivamente.

Na resistência à compressão das composições de concreto leve, o

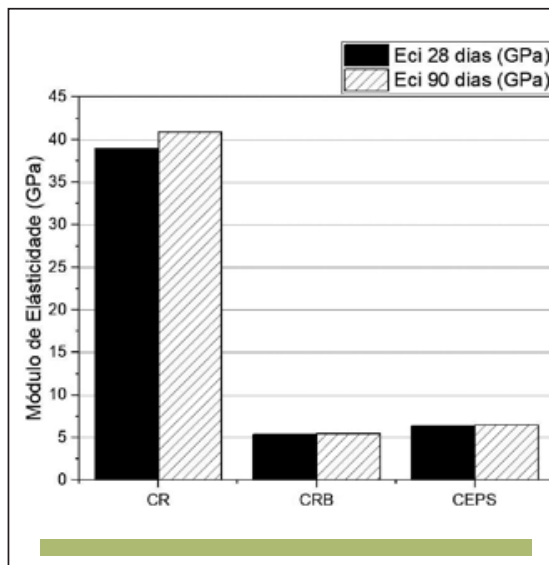

Figura 5

Módulo de elasticidade nas idades de 28 e 90 dias

Fonte: Própria (2021) 


\begin{tabular}{|c|c|c|}
\hline \multicolumn{3}{|c|}{$\begin{array}{l}\text { Tabela } 8 \text { - Resultados dos } \\
\text { concretos a } 28 \text { dias }\end{array}$} \\
\hline Composição & $\begin{array}{l}\text { Absorção } \\
(\%)\end{array}$ & $\begin{array}{c}\text { Índices } \\
\text { de vazios } \\
(\%)\end{array}$ \\
\hline $\mathrm{CR}$ & 3,72 & 8,86 \\
\hline CRBO & 9,5 & 12,24 \\
\hline CEPS & 7,03 & 6,91 \\
\hline \multicolumn{3}{|c|}{ Fonte: Própria (2021) } \\
\hline
\end{tabular}

CEPS apresentou o maior valor, chegando a 9,70 MPa aos 90 dias de cura, enquanto o CRB chegou ao valor de 9,19 MPa aos 90 dias de cura, obtendo valor próximo ao CEPS, mostrando a vantagem do uso de borracha.

Schackow e et al. (2014) em seu estudo o concreto leve, usando apenas $65 \%$ de EPS, obteve resistência de 7,74 MPa, enquanto o valor de todos as composições foram maiores.

Na tração por compressão diametral, a composição de concreto leve que teve o maior valor foi o $\mathrm{CRB}$, chegando a 1,07 MPa de média, enquanto a composição com EPS apresentou o menor valor, chegando a 0,87 MPa de média, mostrando que o resíduo

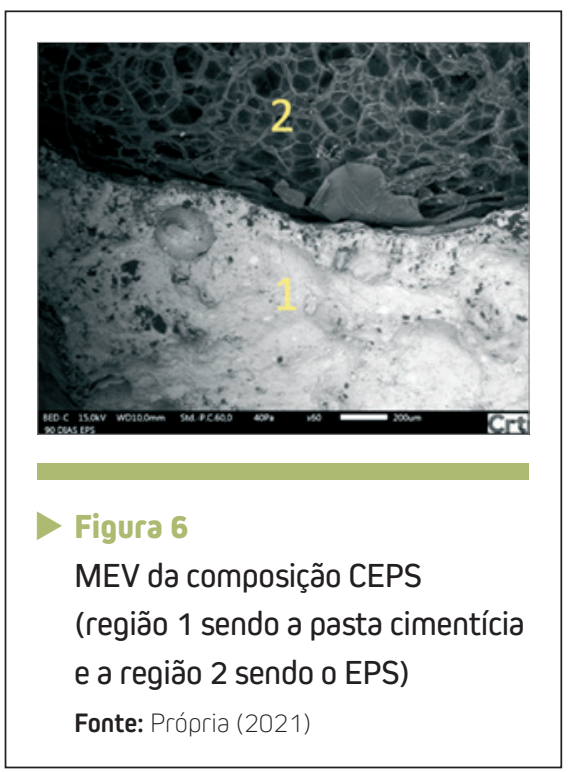

de borracha em comparação ao EPS apresenta melhores resultados na tração por compressão diametral.

O CEPS foi a composição de concreto leve com o maior módulo de elasticidade, com valor de 6,46 GP. Já, o CRB obteve 5,50 GPa. Conclui-se, então, que o resíduo de borracha torna o concreto mais tenaz, corroborando com o estudo de Bekhiti, Ghrieb e Zaitri (2021).

Os resultados da resistência mecânica mostraram que com agregado leve substituindo a brita obtém-se redução significativa, contudo ainda assim as resistências são significativas para aplicações em casos como concreto de regularização, bloco de concreto e entre outros. Em especial, pelo EPS já ser um agregado consolidado no mercado do concreto leve, o uso do resíduo de borracha ter obtido resultados próximos e até levemente superior em algumas das propriedades mecânicas mostra o RB como agregado leve promissor. Uma vez que o CRB obteve resistências superiores a 8 MPa na resistência à compressão axial e 1 MPa na tração por compressão diametral, traz a possibilidade de sua aplicação para regularização, segundo a norma NBR 13281 (ABNT, 2005).

No concreto endurecido, foi ainda realizado o estudo de absorção e índices de vazios. Os ensaios foram realizados com 28 dias de cura. Os resultados obtidos dos ensaios se encontram na Tabela 8.

A absorção e índices de vazios mostraram um leve aumento com o resíduo de borracha, em comparação EPS, uma vez que o EPS é hidrofóbico e, por si só, diminui significativamente a absorção - o fator de apresentar maior índices de vazios e maior absorção corrobora com o fato de o CRB apresentar menor resistência à compressão.

\subsection{Microscopia Eletrônica de varredura (MEV)}

Neste item, foi verificada a ligação dos agregados leves com a pasta de cimento Portland.

A Figura 6 apresenta O MEV do CEPS.

Na Figura 6, identifica-se uma união de certa forma fraca, o que pode ser explicado pela natureza hidrofóbica do EPS.

A Figura 7 apresenta O MEV do CRB.

Na Figura 7, foi possível identificar que o resíduo de borracha apresentou uma boa união com a pasta cimentícia, mostrando uma melhor aderência a matriz do que o EPS.

\section{CONCLUSÕES}

São descartados mais de 1 bilhão de pneus por ano e seu descarte causa muitos problemas sociais e ambientais, o que mostra a necessidade de fazer um reaproveitamento desses resíduos (RB). O estudo de usar esse resíduo na substituição do agregado graúdo em sua totalidade mostrou-se

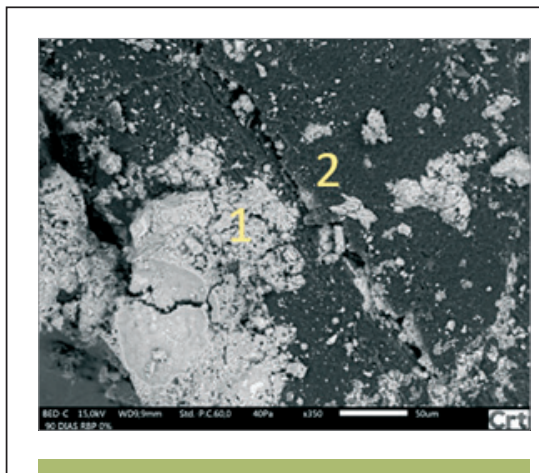

Figura 7

MEV da composição CRB (região 1 sendo a pasta cimentícia e a região 2 sendo o RB)

Fonte: Própria (2021) 
muito eficaz em obter concreto leve. Com o uso do RB foi possível alcançar uma redução da massa específica em mais de $800 \mathrm{~kg} / \mathrm{m}^{3}$, chegando a valores relativamente próximos ao de EPS.
O RB mostrou uma certa deficiência quanto à trabalhabilidade e à segregação da pasta do concreto, corrigidas com uso de superplastificante. Ainda, o RB apresentou redução na proprie- dade mecânica comparado ao concreto convencional, mas, em relação ao concreto leve com pérola de EPS, obteve resultados próximos e, em algumas análises, até superiores.

\section{DREFERÊNCIAS BIBLIOGRÁFICAS}

[1] AMERICAN CONCRETE INSTITUTE - ACI. Standard practice for selecting proportions for structural lightweight concrete, ACl 211.2-98, 2004.

[2] ASSOCIAÇÃO BRASILEIRA DE NORMAS TÉCNICAS. NBR 5738: Concreto - Procedimento para moldagem e cura de corpos-de-prova. Rio de Janeiro, 2015.

[3] BEKHITI, Melik; GHRIEB, Abderrahmane; ZAITRI, Rebih. Effects of tyre rubber fibre and glass fibre on physical and mechanical properties of compressed earth block (C.E.B) based on local materials. Journal of King Saud University - Engineering Sciences, [S.L.], p. 1-12, jan. 2021. Elsevier BV. http://dx.doi.org/10.1016/j. jksues.2021.01.003.

[4] KURZ, Mônica Navarini et al. A potencialidade do uso de resíduo de borracha de pneu em argamassa: análise das propriedades físicas e mecânicas. Matéria (rio de Janeiro), [s.I.], v. 23, n. 3, p.1-20, 18 out. 2018. FapUNIFESP (SciELO).

[5] MOHAJERANI, Abbas et al. Recycling waste rubber tyres in construction materials and associated environmental considerations: a review. : A review. Resources, Conservation And Recycling, [s.I.], v. 155, p. 104679-104696, abr. 2020. Elsevier BV.

[6] SAYADI, A.A.; TAPIA, J.V.; NEITZERT, T.R.; CLIFTON, G.C. Effects of expanded polystyrene (EPS) particles on fire resistance, thermal conductivity and compressive strength of foamed concrete. Revista Elsevier Construction and Building Materials. Vol. 112, pág. 716-724. Amsterdam, PaísesBaixos, 2016.

[7] SCHACKOW, Adilson; EFFTING, Carmeane; FOLGUERAS, Marilena V.; GÜTHS, Saulo; MENDES, Gabriela A.. Mechanical and thermal properties of lightweight concretes with vermiculite and EPS using air-entraining agent. Construction and Building Materials, [S.L.], v. 57, p. 190-197, abr. 2014.

[8] XIE, Yue et al. Preparation and properties of ultra-lightweight EPS concrete based on pre-saturated bentonite. Construction and Building Materials, [s.I.], v. 195 p.505-514, jan. 2019. z

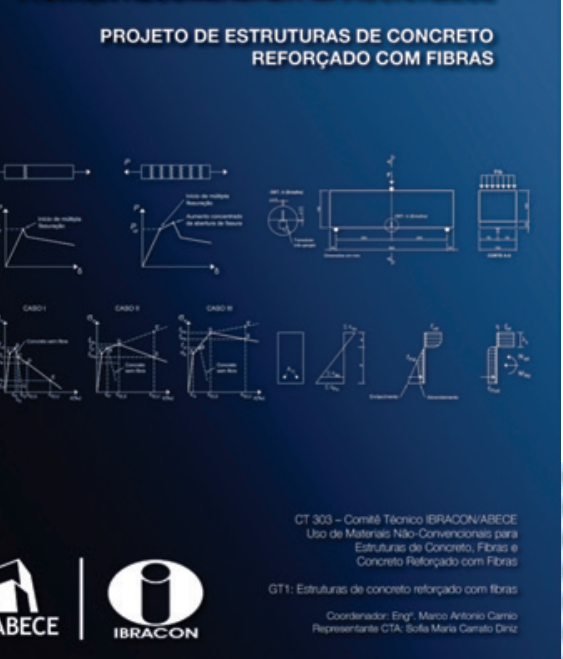

Elaborada pelo CT 303 - Comitê Técnico IBRACON/ABECE sobre Uso de Materiais Não Convencionais para Estruturas de Concreto, Fibras e Concreto Reforçado com Fibras, a Prática Recomendada é um trabalho pioneiro no Brasil, que traz as diretrizes para o desenvolvimento do projeto de estruturas de concreto reforçado com fibras.

Baseada no fib Mode Code 2010, a Prática Recomendada estabelece os requisitos mínimos de desempenho mecânico do CRF para substituição parcial ou total das armaduras convencionais nos elementos estruturais e indica os ensaios para a avaliação do comportamento mecânico do CRF.

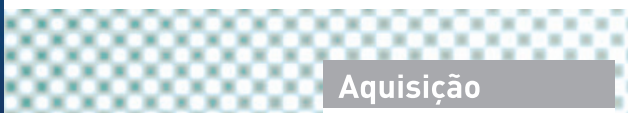

www.ibracon.org.br (loja virtual)

\section{DADOS TÉCNICOS}

ISBN: 978-85-98576-26-8

Edição: $1^{\text {a }}$ edição

Formato: Eletrônico

Páginas: 39

Acabamento: Digital

Ano da publicação: 2016

Coordenador: Eng. Marco Antonio Carnio 